\title{
Association between Breast and Thyroid Cancer among Women in Arkansas
}

\author{
Merry M Peckham, $B A^{1}$, Tyler K Rose, $M S^{1}$, William M Mitchell, $B S^{1}$, Yevgeniya Gokun, $M S^{1}$, \\ Shorabuddin Syed, MS ${ }^{1}$, Deanne King, MD, PhD ${ }^{1}$ and Brendan C Stack, Jr., MD, FACS, FACE ${ }^{1,2^{*}}$
}

${ }^{1}$ University of Arkansas for Medical Sciences, Little Rock, AR, USA

${ }^{2}$ Otolaryngology-HNS, Southern Illinois University School of Medicine, Springfield, IL, USA

\begin{abstract}
Background: Growing evidence suggests a bi-directional relationship between breast and thyroid cancer in women worldwide. This study investigates whether a relationship exists among women in Arkansas.

Methods: Retrospective chart review of patients at University of Arkansas for Medical Sciences was conducted on patients with breast cancer and thyroid disease in The Arkansas Breast Cancer database and EPIC EMR.

Results: Of 12,375 patients with thyroid pathology, 276 had breast cancer, and 40 had thyroid cancer. The relative risks for survivors of one of developing the other were calculated. RR of a breast cancer survivor developing thyroid cancer was $0.826(p=0.3432)$. For the reverse condition, $R R$ was $0.50(p=0.0052)$, which was statistically significant.

Conclusions: Our research suggests that women in Arkansas diagnosed with breast cancer do not have a significant risk of developing thyroid cancer, but that women diagnosed with thyroid cancer are at a reduced risk of developing breast cancer subsequently. Further research is underway to see if these results vary by region in the United States.
\end{abstract}

\section{Introduction}

Breast cancer has the highest rate of new cancer diagnoses and is the second most common cause of cancer-related deaths in the state of Arkansas [1]. Both breast and thyroid cancer rank in the top 10 cancers with the highest rates of incidence in Arkansas and nationwide over the period of 20122016 (Figure 1). Incidence rates are similar between the United and the state of Arkansas, and breast and thyroid cancer rank as 1 and 5, respectively.

Women who are diagnosed with breast cancer routinely undergo surveillance for metastatic disease and may be found incidentally to have thyroid pathology. Recent studies suggest that a link between breast and thyroid cancer exists [2-5]. This trend warrants serious consideration on the part of professional medical societies and clinicians to consider follow up for thyroid nodules in patients already diagnosed with breast cancer. Conversely, the opposite vigilance might apply for breast lumps in thyroid cancer patients [6]. Over the years 2000-2017, the SEER database shows that incidence of breast cancer has remained steady, while the incidence of thyroid cancer has steadily risen (Figure 2).

Interest among this paper's authors on the topic resulted from experience with common clinical scenarios such as the following. A middle-aged female who had been diagnosed and treated for breast cancer would undergo post therapy surveillance for recurrence with whole body 18 FDG Positron emission tomography (PET) scanning. These scans on occasion would show thyroid gland uptake above background levels, which would prompt referral from the breast surgeons to the thyroid surgeons. PET positivity within the thyroid gland would be of 2 types, diffuse or focal [7-10].

Diffuse thyroid uptake on PET is reflective of autoimmune thyroid disease (Hashimoto's Thyroiditis) and is confirmed by thyroid function and antibody testing [11,12]. These patients are then treated appropriately and returned to the care of the breast and primary care teams. Patients with focal thyroid uptake on PET are evaluated according to the latest American Thyroid Association (ATA) guidelines for thyroid nodules [13]. This typically includes thyroid function testing (to rule out hyperthyroidism), ultrasonography of the thyroid and cervical lymphatics, and ultrasound directed fine needle aspiration biopsy for a cytologic diagnosis of the nodule(s) and any lymph-

*Corresponding author: Brendan C Stack, Jr., MD, FACS, FACE, University of Arkansas for Medical Sciences, Little Rock, AR 72205; Otolaryngology-HNS, Southern Illinois University School of Medicine, Springfield, IL 62794, USA

Accepted: October 13, 2020

Published online: October 15, 2020

Citation: Peckham MM, Rose TK, Mitchell WM, et al. (2020) Association between Breast and Thyroid Cancer among Women in Arkansas. J Surgical Endocrinol 2(1):40-45 

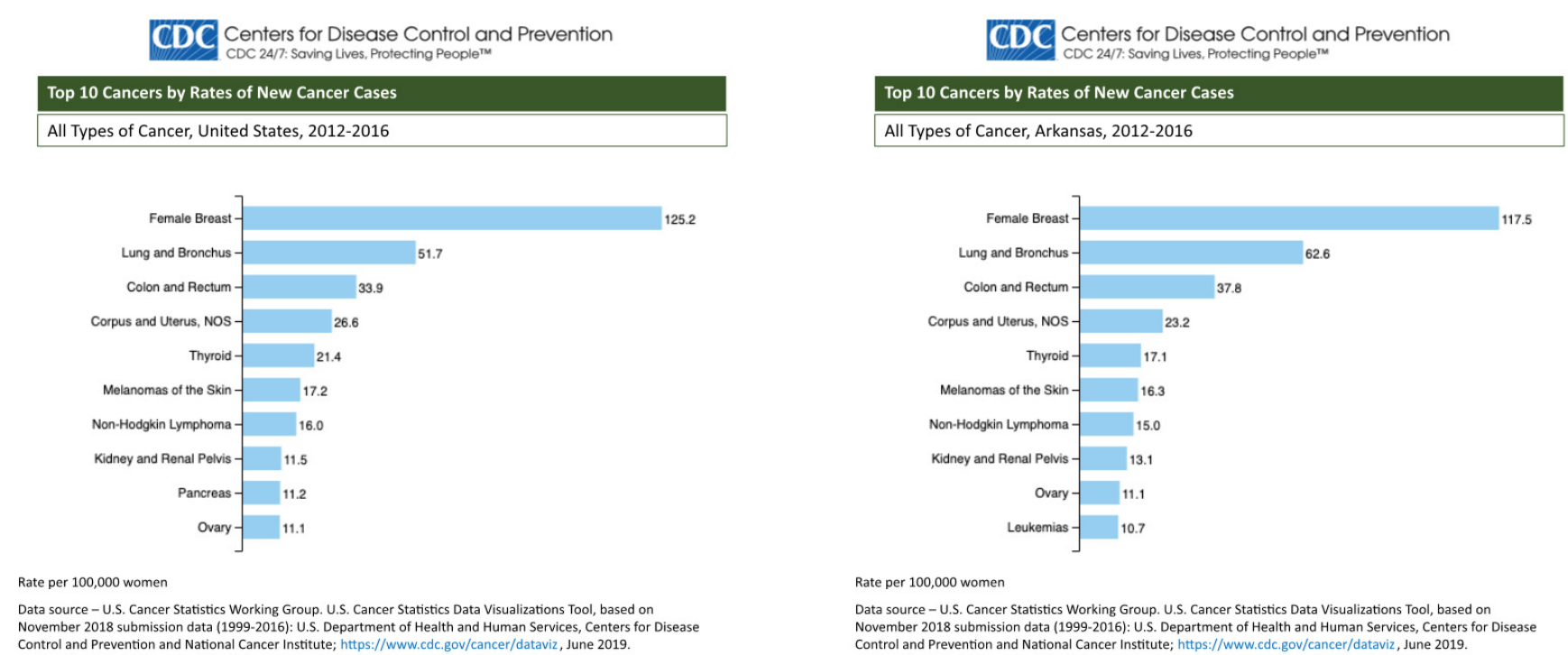

Figure 1: Top 10 cancers by rates of new cancer cases in the United States and Arkansas.

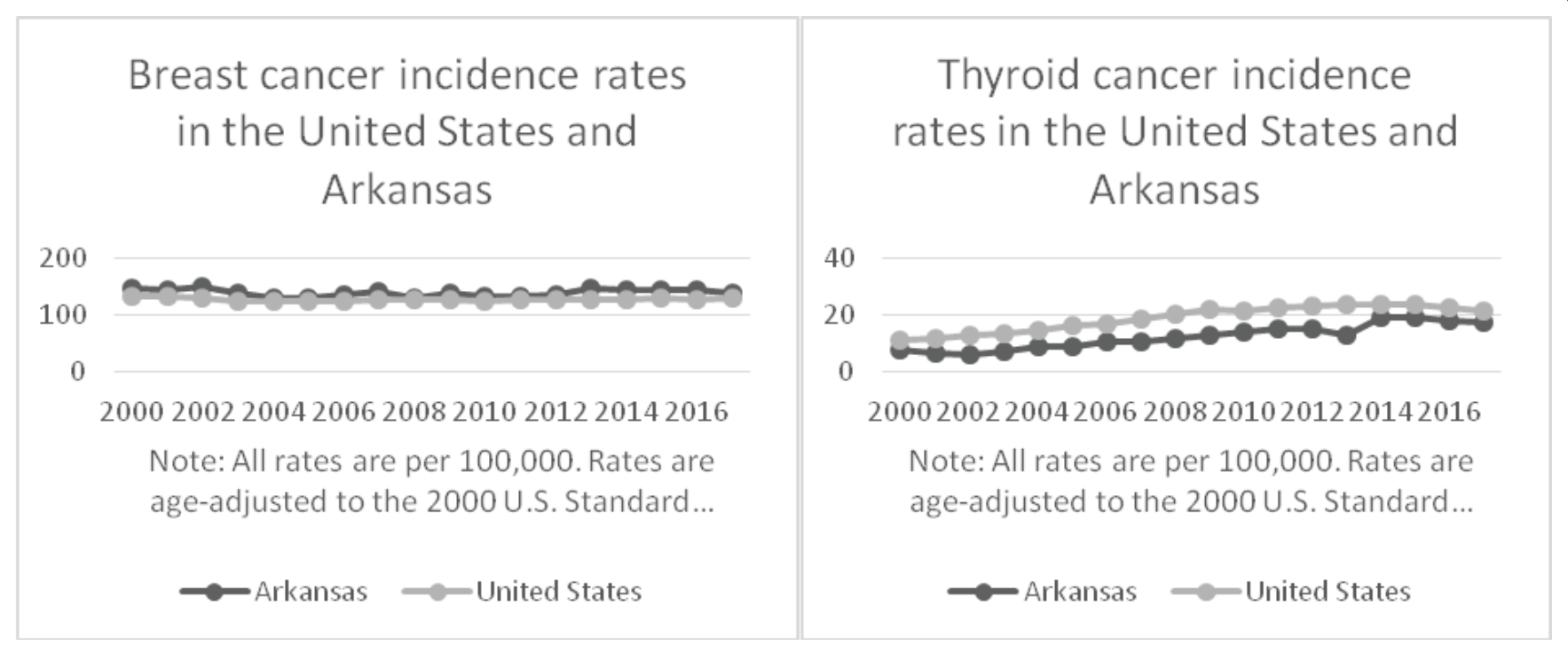

Figure 2: Breast and thyroid cancer incidence rates among women in the Arkansas Cancer Registry and SEER 21 database.

adenopathy which might be present and be of sonographic concern. Cytology results are managed expectantly based on their Bethesda classification and a surgical treatment plan is formulated [14,15].

Based on our interest generated by events in our clinical practice, and a longstanding interest in PET positive thyroid lesions [7-10], we decided to examine our data with respect to this clinical phenomenon. This also raised the question as to whether an absolute prohibition of thyroid nodule screening among current guidelines might need amendment for cases of breast cancer survivors. Therefore, this study examines the association between women diagnosed with breast cancer in Arkansas who also had thyroid pathology, including benign thyroid disease.

\section{Materials and Methods}

The Arkansas Breast Cancer database was cross-referenced with records in UAMS' EPIC EMR, which went live in
May 2014, to identify women who were diagnosed with both breast cancer and thyroid disease as of the date the data was extracted, which was August 6, 2019. A chart review was then conducted to collect dates of diagnosis, histologic subtypes, treatment, patient demographics, and other data on 276 patients with both breast cancer and known thyroid pathology in the EPIC EMR. Of the 276 patients with both breast cancer and thyroid pathology, 2 did not have dates of diagnosis for their breast cancer, and so these cases were not included in the statistical analyses using dates or ages at diagnosis. Upon completion of chart review, descriptive statistics such as means and standard deviations, among others (medians, lower and upper quartiles, minimums and maximums), were calculated for continuous variables such as patients' ages tabulated at breast/thyroid cancer diagnoses. Counts and percents were reported for histologic subtypes of breast and thyroid cancers (categorical variables). Data analyses were conducted using SAS v9/4 (SAS Institute; Cary, NC). The relative 
Citation: Peckham MM, Rose TK, Mitchell WM, et al. (2020) Association between Breast and Thyroid Cancer among Women in Arkansas. J Surgical Endocrinol 2(1):40-45

Table 1: Descriptive statistics.

\begin{tabular}{|l|l|l|l|l|l|l|}
\hline & N & Mean & $\begin{array}{l}\text { Standard } \\
\text { Deviation }\end{array}$ & Median & Minimum & Maximum \\
\hline Age at breast cancer diagnosis & 274 & 56.20 & 12.46 & 57.00 & 24.00 & 87.00 \\
Age at thyroid cancer diagnosis & 40 & 54.13 & 13.72 & 52.00 & 22.00 & 92.00 \\
Time to breast cancer from thyroid cancer & 15 & 8.30 & 7.05 & 7.00 & 0.34 & 25.00 \\
Time to thyroid cancer from breast cancer & 25 & 7.10 & 5.32 & 6.08 & 0.73 & 18.68 \\
\hline
\end{tabular}

Table 2: Breast cancer histology.

\begin{tabular}{|l|l|l|}
\hline & N & Percent \\
\hline Adenocarcinoma & 62 & 22.63 \\
\hline Ductal carcinoma in situ & 33 & 12.04 \\
\hline Infiltrating duct and Lobular carcinoma & 4 & 1.46 \\
\hline Invasive carcinoma of no special type & 18 & 6.57 \\
\hline Invasive ductal carcinoma & 126 & 45.99 \\
\hline Invasive lobular carcinoma & 18 & 6.57 \\
\hline Lobular carcinoma in situ & 3 & 1.09 \\
\hline Mucinous carcinoma & 2 & 0.73 \\
\hline Papillary carcinoma & 2 & 0.73 \\
\hline Phyllodes tumor & 1 & 0.36 \\
\hline Other & 5 & 1.82 \\
\hline Unknown & 2 & Not included \\
\hline Total & 276 & 100 \\
\hline
\end{tabular}

risk (also known as the risk ratio) was also calculated, which is the ratio of the probability of an outcome in an exposed group to the probability of an outcome in an unexposed group. An exposed group in our study is patients with breast cancer diagnosis while unexposed group contains patients without breast cancer diagnosis. The outcome of interest is thyroid cancer diagnosis. The relative risk was calculated using PROC FREQ with option of "relrisk" using SAS v9.4 software.

\section{Results and Discussion}

\section{Results}

There were 264,887 women who had an appointment with an otolaryngologist found in the EPIC data source since May 2014. Based on this sample, there were 5,645 women who had a diagnosis of breast cancer and 1,414 women who had a diagnosis of thyroid cancer during the same time period. Out of 5,645 women with a diagnosis of breast cancer, $25(0.44 \%)$ women also had thyroid cancer diagnosed after breast cancer. Out of the remaining 259,242 women without a breast cancer diagnosis, $1,389(0.54 \%)$ women were diagnosed with thyroid cancer. The average age at breast cancer diagnosis among 274 women was 56-years-old, while the average age at thyroid cancer diagnosis was 54 -years-old. The most common histologic subtype of breast cancer among 276 women was invasive ductal carcinoma (46\%), and more than two-thirds (68\%) of women had papillary thyroid cancer histology.

The women in this study were at a $17 \%$ lower risk of developing thyroid cancer after breast cancer but this relationship
Table 3: Thyroid cancer histology.

\begin{tabular}{|l|l|l|}
\hline & N & Percent \\
\hline Follicular & 3 & 7.89 \\
\hline Medullary & 2 & 5.26 \\
\hline Papillary & 26 & 68.42 \\
\hline Other & 7 & 31.58 \\
\hline Unknown & 2 & Not included \\
\hline Total & 40 & 100 \\
\hline
\end{tabular}

Table 4: Race and ethnicity.

\begin{tabular}{|l|l|l|}
\hline & N & Percent \\
\hline Black & 6 & 15.00 \\
\hline White & 34 & 85.00 \\
\hline Total & 40 & 100 \\
\hline
\end{tabular}

was not statistically significant (RR: $0.83 ; 95 \% \mathrm{Cl}: 0.56-1.23$ ). Women who had already had a diagnosis of thyroid cancer significantly reduced the risk by half of developing subsequent diagnosis of breast cancer compared to those women without history of thyroid cancer (RR: $0.50 ; 95 \% \mathrm{Cl}$ : $0.30-$ 0.83 ). Descriptive statistics for both groups are summarized in Table 1. Histologic subtypes for breast cancer are given in Table 2, and subtypes for thyroid cancer are given in Table 3. All patients were female, and demographics for race and ethnicity are given in Table 4.

\section{Discussion}

Cancer, in general, is understood to be a disease with a pathogenesis that is fundamentally genetic, whether it arises from damage to DNA (inherited or environmentally caused) in individual cells, or from missing or defective gene copies or gene products (proteins) that regulate expression or control cell proliferation. As such, researchers increasingly find that certain types of cancers tend to occur in clusters. Examples include Lynch syndrome, BRCA1 and BRCA2, MEN syndromes, and many others [16].

The clinical scenario outlined in the introduction of this paper occurred often enough to prompt the authors to take notice and ask whether thyroid and breast cancer share an association that may be rooted in a common etiology. If such an association were found to exist, it would impact recommendations on screening breast cancer survivors for thyroid cancer. Additionally, establishment of any associations would alert clinicians to risks that need to be considered in the care of these cancer patients.

As treatment for cancer has improved, more patients are 
surviving to develop recurrence and second primary cancers [17-23]. In 2006, the Institute of Medicine called for the creation of survivorship care plans for cancer survivors to address these patients growing long-term care needs [23]. The Childhood Oncology Group (COG) and American Cancer Society (ACS) responded by providing evidence-based guidelines for clinicians to refer to in creating these plans for their patients [17-21,24]. The COG guidelines recommend screening for second primaries in survivors of childhood, adolescent, and young adult cancers, specifically for breast and colorectal cancers [24]. The ACS developed survivorship guidelines for 4 primary cancers, namely breast, colorectal, prostate, and head and neck cancer (HNC) [18-21]. HNC included cancers caused by HPV or smoking, not the thyroid [21], and this set of survivors was the only group for whom the ACS considered the primary cancer a factor that placed them at greater risk than the general public. For HNC survivors, the ACS recommends additional screening for lung cancer [21].

For all other cancer survivors, ACS guidelines state that those with average risk undergoes the same screening for second primary cancers (SPC) as the general public [17-20]. Patients with high risk for cancers are recommended to undergo additional imaging [17-20]. Per the ACS, breast cancer survivors are considered high risk if they have a strong family history of breast cancer, a genetic mutation such as BRCA1 or BRCA2, or if they received radiation therapy for another cancer, such as Hodgkin lymphoma [25]. Yet there is a growing body of evidence that suggests these guidelines may be incomplete, and that clinicians should have a higher level of suspicion that their breast cancer patient may have, or may yet develop, a second primary thyroid malignancy $[2-5,26]$.

A higher than expected incidence of thyroid cancer has been observed in breast cancer patients. One recent meta-analysis reports an odds ratio (OR) of a female developing thyroid cancer after a breast cancer to be 1.55 (expected OR 1.0 in the general population) [2]. With this increased incidence having been observed, many have begun to speculate on the impact that routine thyroid cancer screening would have on this select patient population [2,27-29]. Currently, both the US Preventive Task Force [30] and the ATA [13] do not recommend routine thyroid cancer screening in any population other than those defined as high-risk, which includes only patients with a history of radiation exposure or a family history of differentiated thyroid cancer.

Nielsen, et al.'s meta-analysis found a significantly elevated odds ratio for patients diagnosed with either cancer of developing the other, but these studies did not measure how much time passed between diagnoses [2]. Similarly, Dong, et al. and Bolf, et al. published studies examining possible etiologies for bi-directional synchronous and metachronous thyroid and breast cancer, each noting that additional studies are warranted to better understand how the two cancers are related $[4,26]$. Huang, et al. studied 13,978 patients with breast cancer and examined how many of them developed thyroid cancer, as well as when and of what histology were both cancers [3]. Their results showed that $17.4 \%$ of patients had their thyroid cancer diagnosed more than a year before their diagnosis of breast cancer, $30.0 \%$ had synchronous di- agnosis, defined as within one year of each other, and $62.6 \%$ were diagnosed with thyroid cancer at least a year after their diagnosis of breast cancer. They concluded that there may be different mechanisms underlying synchronous and metachronous disease [3].

Considering the reverse case (thyroid cancer preceding breast cancer), there has also been interest in studying whether there is an increased risk of thyroid cancer survivors developing breast cancer [31-33]. In 2001, a single-center analysis published by Chen, et al. found a relative risk of 1.42 for developing breast cancer in thyroid cancer survivors [31]. A meta-analysis was published subsequently in 2007 by Subramanian, et al. in Thyroid that found a standardized incidence ratio (SIR) of 1.25 for thyroid cancer survivors of developing breast cancer [32]. Kim, et al. further found a SIR of 1.13 in their cohort and, importantly, that elevated risk was associated with a smaller tumor size [33].

The results observed in our study indicate that clinicians may reassure their patients if a PET-positive nodule is found during workup of breast cancer that no relationship between the two cancers has been found among women in the state of Arkansas. The recommendations set forth by the ATA [13] are sufficient for women in our state. If the patient hails from outside Arkansas, consider that recent research suggests an increased risk of developing thyroid cancer as a SPC.

Limitations of our study include how the data was obtained and how both breast and thyroid cancer have been diagnosed and treated over the extended period under consideration. As outlined in the introduction, a common scenario that prompts workup for thyroid cancer in patients with breast cancer is increased uptake on PET. However, the use of PET scans was not introduced as routine screening for metastatic disease in breast cancer until the late 1990's [15,34], therefore many women may have developed clinical thyroid pathology that was not found earlier because they were never given a PET scan. Further, advancements in treatment of breast cancer have increased survivorship among women $[17,18]$, and it is possible that women who would have developed thyroid cancer might have succumbed to their breast cancer prior to the possibility of developing a thyroid cancer if they were treated in the distant past [35].

The data for this project was extracted from the EPIC EMR, which was implemented in 2014 at UAMS. Owing to the large number of patients flagged with a diagnosis of thyroid pathology, a chart review was conducted only on patients who had both thyroid pathology and breast cancer. During the chart review process, the reviewer often relied on physician notes or reported patient recollection of dates of diagnosis and interventions. As a result, documentation of thyroid or breast pathology in these records may be inaccurate. For example, the specific type of pathology and date of diagnosis was not always recorded. Sometimes only the month or year of diagnosis was available, and receptor status for the breast cancer was sometimes incomplete or not recorded.

\section{Conclusions}

Among the patients in our study, there was no increased risk of developing either cancer in women in Arkansas. How- 
Citation: Peckham MM, Rose TK, Mitchell WM, et al. (2020) Association between Breast and Thyroid Cancer among Women in Arkansas. J Surgical Endocrinol 2(1):40-45

ever, among women who did develop thyroid cancer after breast cancer, a positive family history was only found in 2 women, and of those 1 was diagnosed with thyroid cancer after breast cancer. The significant finding of a RR of 0.5 for women with thyroid cancer of developing breast cancer subsequently is surprising. These results may be valid exclusively for the state of Arkansas. More work needs to be done to assess whether there are regional differences in these relative risks, which is currently being undertaken.

Whether they can be further generalised to the nation is under investigation currently in collaboration with other institutions through the Accrual to Clinical Trials (ACT) network, with the cooperation of: University of California - Davis, University of California - Irvine, University of Texas Health Science Center at San Antonio, University of Kansas Medical Center, University of Alabama - Birmingham, Pennsylvania State University, University of California - San Francisco, Emory University, and University of Kentucky.

\section{Data Availability}

Data for Figures 1 and 2:

U.S. Cancer Statistics Working Group. U.S. Cancer Statistics Data Visualizations Tool, based on 2019 submission data (1999-2017): U.S. Department of Health and Human Services, Centers for Disease Control and Prevention and National Cancer Institute; www.cdc.gov/cancer/dataviz, released in June 2020.

Surveillance, Epidemiology, and End Results (SEER) Program (www.seer.cancer.gov) SEER*Stat Database: Incidence - SEER Research Data, 9 Registries, Nov 2019 Sub (1975-2017) - Linked To County Attributes - Time Dependent (1990-2017) Income/Rurality, 1969-2017 Counties, National Cancer Institute, DCCPS, Surveillance Research Program, released April 2020, based on the November 2019 submission.

Age-Adjusted Cancer Incidence Rates in Arkansas, 2000 2017. Based on data released May 2020. Cancer-Rates.info. Accessed on Jun 08, 2020. Arkansas Cancer Registry. <http:// cancer-rates.info/ar/>

Data from our study is available upon request. Contact $\mathrm{Dr}$. Deanne King at DKing3@uams.edu.

\section{Conflicts of Interest}

The author(s) declare(s) that there is no conflict of interest regarding the publication of this paper.

\section{Funding Statement}

No funding.

\section{Acknowledgments}

None.

\section{Supplementary Materials}

None.

\section{References}

1. U.S. Cancer Statistics Working Group. U.S. Cancer Statistics Data
Visualizations Tool, based on November 2018 submission data (1999-2017) U.S. Department of Health and Human Services, Centers for Disease Control and Prevention and National Cancer Institute.

2. Nielsen SM, White MG, Hong S, et al. (2016) The breast-thyroid cancer link: A systematic review and meta-analysis. Cancer Epidemiol Biomarkers Prev 25: 231-238.

3. Huang NS, Chen XX, Wei WJ, et al. (2018) Association between breast cancer and thyroid cancer: A study based on 13978 patients with breast cancer. Cancer Med 7: 6393-6400.

4. Dong L, Lu J, Zhao B, et al. (2018) Review of the possible association between thyroid and breast carcinoma. World J Surg Oncol 16: 130.

5. Joseph KR, Edirimanne S, Eslick GD (2015) The association between breast cancer and thyroid cancer: A meta-analysis. Breast Cancer Res Treat 152: 173-181.

6. American Cancer Society (2019) Cancer facts \& figures 2019. American Cancer Society, Atlanta.

7. Stack BC Jr, Bodenner DL, Bartel TB, et al. (2012) Re: Thyroid incidentalomas in FDG-PET/CT: Prevalence and clinical impact. Eur Arch Otorhinolaryngol 269: 1873.

8. Boeckmann J, Bartel T, Siegel E, et al. (2012) Can the pathology of a thyroid nodule be determined by positron emission tomography uptake? Otolaryngol Head Neck Surg 146: 906-912.

9. Rothman IN, Middleton L, Stack BC Jr, et al. (2011) Incidence of diffuse FDG uptake in the thyroid of patients with hypothyroidism. Eur Arch Otorhinolaryngol 268: 1501-1504.

10. King DL, Stack BC Jr, Spring PM, et al. (2007) Incidence of thyroid carcinoma in fluorodeoxyglucose positron emission tomography-positive thyroid incidentalomas. Otolaryngol Head Neck Surg 137: 400-404.

11. Karantanis D, Bogsrud TV, Wiseman GA, et al. (2007) Clinical significance of diffusely increased $18 \mathrm{~F}-\mathrm{FDG}$ uptake in the thyroid gland. Journal of Nuclear Medicine 48: 896-901.

12. Akamizu T, Amino N (2017) Hashimoto's thyroiditis. In: Feingold KR, Anawalt B, Boyce A, et al., Endotext. MDText.com, Inc. South Dartmouth (MA).

13. Haugen BR, Alexander EK, Bible KC, et al. (2016) 2015 American thyroid association management guidelines for adult patients with thyroid nodules and differentiated thyroid cancer: The American thyroid association guidelines task force on thyroid nodules and differentiated thyroid cancer. Thyroid 26: 1-133.

14. Tamhane S, Gharib H (2016) Thyroid nodule update on diagnosis and management. Clinical Diabetes and Endocrinology 2: 17.

15. Delbeke D, Coleman RE, Guiberteau MJ, et al. (2006) Procedure guideline for tumor imaging with 18F-FDG PET/CT 1.0. Journal of Nuclear Medicine 47: 885-895.

16. Massingham L, De Souza A (2013) Current indications for consideration of evaluation for hereditary cancer predisposition syndromes and how they can change management. R I Med J 103: $20-24$.

17. De Angelis R, Sant M, Coleman MP, et al. (2014) Cancer survival in Europe 1999-2007 by country and age: Results of EUROCARE: 5-a population-based study. Lancet Oncol 15: 23-34.

18. Runowicz CD, Leach CR, Henry NL, et al. (2016) American cancer society/American society of clinical oncology breast cancer survivorship care guideline. CA Cancer J Clin 66: 43-73. 
Citation: Peckham MM, Rose TK, Mitchell WM, et al. (2020) Association between Breast and Thyroid Cancer among Women in Arkansas. J Surgical Endocrinol 2(1):40-45

19. El-Shami K, Oeffinger KC, Erb NL, et al. (2015) American cancer society colorectal cancer survivorship care guidelines. CA Cancer J Clin 65: 428-455.

20. Skolarus TA, Wolf AM, Erb NL, et al. (2014) American cancer society prostate cancer survivorship care guidelines. CA Cancer J Clin 64: 225-249.

21. Cohen EE, LaMonte SJ, Erb NL, et al. (2016) American cancer society head and neck cancer survivorship care guideline. CA Cancer J Clin 66: 203-239.

22. Soliman SE, Racher H, Zhang C, et al. (2017) Genetics and molecular diagnostics in retinoblastoma--an update. Asia Pac J Ophthalmol (Phila) 6: 197-207.

23. Institute of Medicine (2005) From cancer patient to cancer survivor: Lost in transition. National Academies Press, Washington, DC.

24. Landier W, Bhatia S, Eshelman DA, et al. (2004) Development of risk-based guidelines for pediatric cancer survivors: The children's oncology group long-term follow-up guidelines from the children's oncology group late effects committee and nursing discipline. J Clin Oncol 22: 4979-4990.

25. Saslow D, Boetes C, Burke W, et al. (2007) American Cancer Society guidelines for breast screening with $\mathrm{MRI}$ as an adjunct to mammography. CA Cancer J Clin 57: 75-89.

26. Bolf EL, Sprague BL, Carr FE (2019) A linkage between thyroid and breast cancer: A common etiology? Cancer Epidemiol Biomarkers Prev 28: 643-649.
27. Lee HK, Hur MH, Ahn SM (2003) Diagnosis of occult thyroid carcinoma by ultrasonography. Yonsei Medical Journal 44: 1040-1044.

28. Park JS, Oh KK, Kim EK, et al. (2007) Sonographic detection of thyroid cancer in breast cancer patients. Yonsei Medical Journal 48: 63-68.

29. Kim SS, Kim SJ, Bae YT, et al. (2012) Factors associated with the development of new onset diffuse thyroid F18-fluorodeoxyglucose uptake after treatment of breast cancer in patients without a history of thyroid disease or thyroid dysfunction. Thyroid 22: 53-58.

30. US Preventive Services Task Force, Bibbins-Domingo K, Grossman DC, et al. (2017) Screening for thyroid cancer: US preventive services task force recommendation statement. JAMA 317: 1882-1887.

31. Chen AY, Levy L, Goepfert H, et al. (2001) The development of breast carcinoma in women with thyroid carcinoma. Cancer 92: 225-231.

32. Subramanian S, Goldstein DP, Parlea L, et al. (2007) Second primary malignancy risk in thyroid cancer survivors: A systematic review and meta-analysis. Thyroid 17: 1277-1288.

33. Kim C, Bi X, Pan D, et al. (2013) The risk of second cancers after diagnosis of primary thyroid cancer is elevated in thyroid microcarcinomas. Thyroid 23: 575-582.

34. Yang SK, Cho N, Moon WK (2007) The role of PET/CT for evaluating breast cancer. Korean J Radiol 8: 429-437.

35. Cibas ES, Ali SZ (2017) The 2017 bethesda system for reporting thyroid cytopathology. Thyroid 27: 1341-1346.

DOI: $10.36959 / 608 / 445$

Copyright: (C) 2020 Niazi S. This is an open-access article distributed under the terms of the Creative Commons Attribution License, which permits unrestricted use, distribution, and reproduction in any medium, provided the original author and source are credited. 